\title{
Translational repression of HIF2 $\alpha$ expression in mice with Chuvash polycythemia reverses polycythemia
}

\author{
Manik C. Ghosh, ${ }^{1}$ De-Liang Zhang, ${ }^{1}$ Hayden Ollivierre, ${ }^{1}$ Michael A. Eckhaus, ${ }^{2}$ and Tracey A. Rouault $^{1}$ \\ ${ }^{1}$ Metals Biology and Molecular Medicine Group, Eunice Kennedy Shriver National Institute of Child Health and Human Development (NICHD), and ${ }^{2}$ Veterinary Resources Program, NIH, Bethesda, Maryland, USA.
}

\begin{abstract}
Chuvash polycythemia is an inherited disease caused by a homozygous germline $V H L^{R 200 w}$ mutation, which leads to impaired degradation of HIF2 $\alpha$, elevated levels of serum erythropoietin, and erythrocytosis/polycythemia. This phenotype is recapitulated by a mouse model bearing a homozygous VhI ${ }^{2200 w}$ mutation. We previously showed that iron-regulatory protein 1-knockout (Irp1-knockout) mice developed erythrocytosis/polycythemia through translational derepression of Hif2 $\alpha$, suggesting that IRP1 could be a therapeutic target to treat Chuvash polycythemia. Here, we fed Vh/R20ow mice supplemented with Tempol, a small, stable nitroxide molecule and observed that Tempol decreased erythropoietin production, corrected splenomegaly, normalized hematocrit levels, and increased the lifespans of these mice. We attribute the reversal of erythrocytosis/polycythemia to translational repression of Hif2 $\alpha$ expression by Tempol-mediated increases in the IRE-binding activity of Irp1, as reversal of polycythemia was abrogated in Vh/R200w mice in which Irp1 was genetically ablated. Thus, a new approach to the treatment of patients with Chuvash polycythemia may include dietary supplementation of Tempol, which decreased Hif2 $\alpha$ expression and markedly reduced life-threatening erythrocytosis/polycythemia in the Vh/R20ow mice.
\end{abstract}

\section{Introduction}

Chuvash polycythemia is an autosomal recessive disorder that is endemic to the Chuvash population of the Russian Federation (1) and the Italian island of Ischia, but has also been found in patients around the world (2). Patients with Chuvash polycythemia die prematurely as a result of complications including thrombosis and cerebral hemorrhage (3). The major symptoms and signs found in these patients are headache, fatigue, abdominal pain, chest pain, plethora, hepatomegaly, and clubbing (3). Chuvash polycythemia is caused by a homozygous mutation in the von Hippel-Lindau (VHL) tumor-suppressor gene that results in a R200W amino acid substitution.

The VHL gene is involved in the hypoxia-sensing pathway, and it encodes a multifunctional protein that interacts with elongin $\mathrm{B}$, elongin $\mathrm{C}$, cullin-2, and RING-box protein 1 (Rbx1) in a complex referred to as VCB-CUL2. This VCB-CUL2 complex functions as a ubiquitin ligase that promotes the degradation of $\alpha$-subunits of HIFs $(4,5)$. More than 1,500 germline and somatic mutations have been identified in the VHL gene. All of these mutations cause VHL disease, except the 3 missense mutations $V H L^{R 200 w}, V H L^{H 191 D}$, and $V H L^{P 138 L}(6-8)$. These 3 homozygous mutations in the $V H L$ gene cause congenital erythrocytosis/polycythemia, whereas patients with VHL disease develop vascularized tumors, including clearcell renal cell carcinomas (CCRCCs), cerebral hemangioblastoma, pheochromocytoma, endolymphatic sac tumors (ELSTs), epididymal or broad ligament cystadenomas, and pancreatic tumors $(6,9)$.

The VHL protein targets the $\alpha$ subunits of hypoxia-inducible factors (HIF1 $\alpha$ and HIF2 $\alpha$ ) for proteasomal degradation after prolyl hydroxylase domain protein 2 (PHD2) site-specifically hydroxylates

Conflict of interest: The authors have declared that no conflict of interest exists

Submitted: September 27, 2017; Accepted: January 9, 2018.

Reference information: J Clin Invest. 2018;128(4):1317-1325.

https://doi.org/10.1172/JCI97684.
$\mathrm{HIF} \alpha$ proteins at normal oxygen concentrations. However, under low oxygen (hypoxic) conditions or when there is a loss of VHL function, HIF1 $\alpha$ and HIF2 $\alpha$ (also known as endothelial PAS domain protein 1 [EPAS1]) proteins are stabilized. Since VHL is required for the degradation of HIF $\alpha$ proteins (10), and its dysfunction promotes the transcription of multiple HIF $\alpha$ targets, including erythropoietin (EPO), a glycoprotein hormone essential for erythropoiesis (11), Chuvash polycythemia is associated with elevated levels of hypoxia-inducible factors HIF1 $\alpha$ and HIF2 $\alpha$ and higher levels of serum erythropoietin $(12,13)$. A mouse model of Chuvash polycythemia bearing the same homozygous $V h l^{R 200 W}$ mutation that has been detected in patients with Chuvash polycythemia recapitulated the erythrocytosis/polycythemia phenotype by manifesting increased hematocrit, RBC, and hemoglobin levels and slightly elevated levels of serum erythropoietin and Hif $2 \alpha(14)$.

Iron is intimately associated with oxygen sensing in cells (15), and both erythropoiesis and proteasomal degradation of HIF $2 \alpha$ are regulated by iron. Two cytosolic iron regulatory proteins, IRP1 and IRP2, regulate many aspects of cellular iron homeostasis. IRPs posttranscriptionally control the expression of transcripts that contain iron-responsive element (IRE) sequences of iron metabolism proteins, including the iron import proteins transferrin receptor 1 (TfR1) and divalent metal ion transporter 1 (DMT1), the iron export protein ferroportin, the iron storage protein ferritin, and hypoxia-inducible factor $2 \alpha$ (HIF2 $\alpha$ ). IRP1 exists in a dynamic equilibrium between 2 forms: a cytosolic aconitase form with a $4 \mathrm{Fe}-4 \mathrm{~S}$ cluster in its active site and an apoprotein form that displays IRE-binding activity. When cellular iron levels are low, IRP1 exists predominantly in the IRE-binding form that lacks an intact Fe-S cluster. When cellular iron levels are high, IRP1 regains its iron-sulfur cluster and exists mainly in the cytosolic aconitase form. The cytosolic aconitase form is also stabilized at low oxygen concentrations $(16,17)$. 

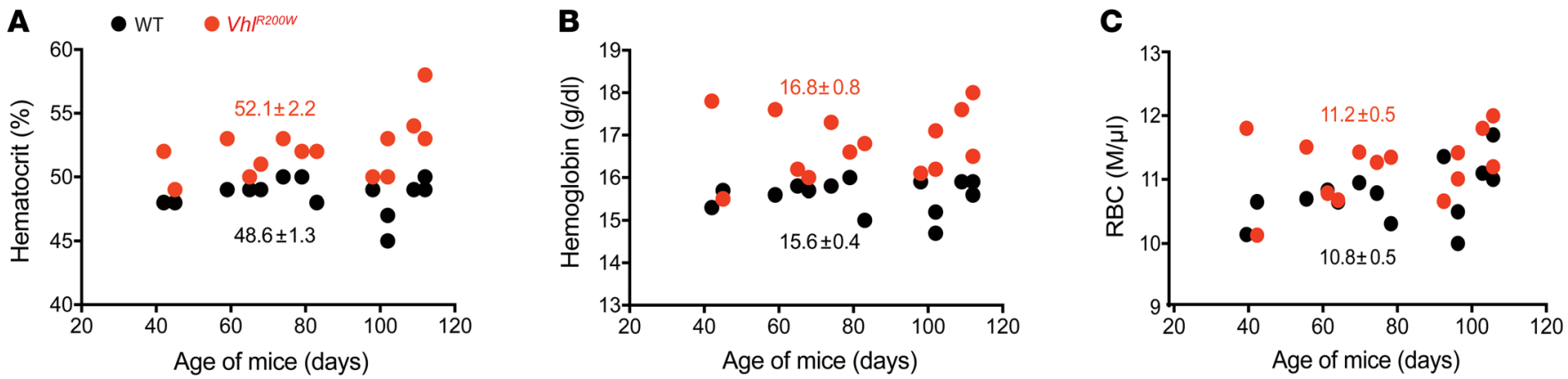

Figure 1. Vh/R20ow mice developed erythrocytosis/polycythemia at early ages. (A) Hematocrit levels as determined by capillary tube centrifugation, (B) hemoglobin levels, and (C) RBC counts of Vh/R20ow mice (red circles) were significantly higher than those of their WT littermates (black circles) as early as 7 weeks of age. $(\mathbf{A}-\mathbf{C}) P<0.0001$. To determine the $P$ values, statistical analyses were performed using a 2 -tailed paired $t$ test. The mean \pm $\mathrm{SD}$ is indicated in each panel.

Recently, we and others discovered that mice that lack Irp1 develop erythrocytosis/polycythemia (18-20). Hif2 $\alpha$, but not HIF1 $\alpha$, has an IRE in the $5^{\prime}$-UTR of its mRNA, and binding of Irp1 to this Hif $2 \alpha$ IRE enables Irp1 to repress the translation of Hif $2 \alpha$ and thus reduce the production of erythropoietin (18-21). Therefore, deletion of Irp1 causes erythrocytosis/polycythemia in mice through translational derepression of Hif $2 \alpha$. Hence, activation of Hif $2 \alpha$ IRE binding of Irp1 is expected to reduce erythropoiesis. Here, we used Tempol (4-hydroxy-2,2,6,6-tetramethylpiperidine- $N$-oxyl), a commercially available stable nitroxide radical that can readily permeate biological membranes $(22,23)$, to increase the IRE-binding activity of Irp1. Tempol has been shown to produce beneficial effects in cell and animal models of tumorigenesis, shock, hypertension, diabetes, ischemia-reperfusion injury, traumatic brain injury, and Parkinson's disease (24-27) as well as to prevent alopecia induced by whole-brain radiotherapy in metastatic cancer patients when applied topically to the scalp (28). We previously showed that Tempol disassembles the cytosolic iron sulfur cluster of Irp1 and consequently activates its latent IRE-binding activity $(29,30)$. Combining these two unique molecular pathways of modulation of IRE-binding activity of Irp1 by Tempol and translational regulation of Hif $2 \alpha$ by Irp1, we hypothesized that activation of Hif $2 \alpha$ IRE binding of Irp1 in polycythemic $V h l^{R 200 W}$ mice by Tempol would inhibit HIF $2 \alpha$ translation, reduce erythropoietin levels, and correct erythrocytosis/polycythemia.

\section{Results}

Tempol reverses plethora, splenomegaly, and erythrocytosis/polycythemia in Vhl ${ }^{R 200 W_{-}}$mutant mice. Erythrocytosis/polycythemia develops as early as 2 to 4 years of age in patients with Chuvash polycythemia (3). To elucidate whether $V h l^{R 200 W}$ mice develop erythrocytosis/polycythemia at such early ages, we measured the hematocrit levels of WT and R200W-mutant littermates at the ages of 42 to 112 days by capillary centrifugations as well as with a hematology analyzer, and we observed that the development of erythrocytosis/polycythemia started in $V h l^{R 200 W}$ mice at as early as 6 weeks of age (Figure 1A and Supplemental Figure 1; supplemental material available online with this article; https://doi.org/10.1172/ JCI97684DS1), comparable to the temporal onset seen in very young human patients. The mutant mice also showed increased hemoglobin levels and RBC counts at early ages (Figure 1, B and C).
Vhl ${ }^{R 200 W}$ mice developed erythema in their paws and snouts (Figure 2A) as previously observed (14), analogous to the plethora seen in human patients with polycythemia. When these mutant mice were placed on a Tempol-supplemented diet at 6 to 8 weeks of age for a period of 3 to 6 months, the erythema in the paws and snouts disappeared, and they looked more like the WT animals. The spleens of $V h l^{R 200 W}$ mice were enlarged compared with those of their WT littermates (Figure 2B), indicating that splenomegaly and extramedullary erythropoiesis developed in these mutant mice. However, the spleens of $V h l^{R 20 o w}$ mice fed a Tempol-supplemented diet looked normal and more like those of WT animals, suggesting that Tempol treatment reversed splenomegaly in these mutant mice.

We measured hematocrit levels of WT and $V h l^{R 200 W}$ mice maintained either on a control diet or on the same diet supplemented with Tempol and observed a significant increase in the hematocrit levels of control diet-fed $V h l^{R 200 W}$ mice in comparison with levels of WT mice on a control diet (Figure 2, C and D, and Supplemental Figure 2A), reconfirming that these mice had erythrocytosis/polycythemia. In addition, hemoglobin levels and RBC counts also significantly increased in the control diet-fed $V h l^{R 200 W}$ mice (Figure 2, E and F), as was observed earlier (14). Importantly, with Tempol treatment, the hematocrit, hemoglobin, and RBC levels of $V h l^{R 200 W}$ mice decreased significantly (Figure 2, C-F), consistent with our conclusion that erythropoiesis was reduced in Tempol-supplemented mutant mice. We did not observe any significant effect of either the mutation or the diet on RBC indices, reticulocytes, WBC counts, platelet counts, or serum glucose levels in $V h l^{R 200 W}$ mice (Supplemental Figure 2, B-H). We measured serum ferritin levels in control diet- and Tempol diet-fed WT and $V h l^{R 200 W}$ mice using the ELISA method and observed that serum ferritin levels were slightly but significantly higher in the control diet-fed $V h l^{R 200 W}$ mice compared with levels in WT mice (Supplemental Figure 2I), perhaps due to macrophage iron overload as a result of increased erythrophagocytosis, as macrophages are the main source of serum ferritin (31).

Decreased erythropoiesis in Tempol-treated Vhl-R200W mice results from the downregulation of EPO production caused by translational repression of Hif $2 \alpha$. An earlier study showed that patients with Chuvash polycythemia had significantly higher serum erythropoietin concentrations (3). In previous work with $V h l^{R 200 w}$ mice (14), the researchers observed increased serum EPO, but the difference rela- 
A

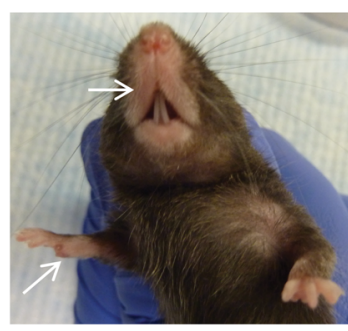

WT Control diet

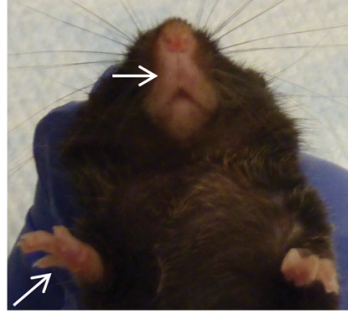

Vh/R200w Control diet

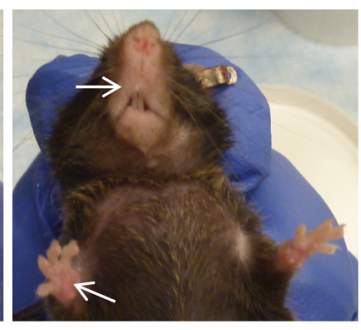

Vh/R20ow Tempol diet
B
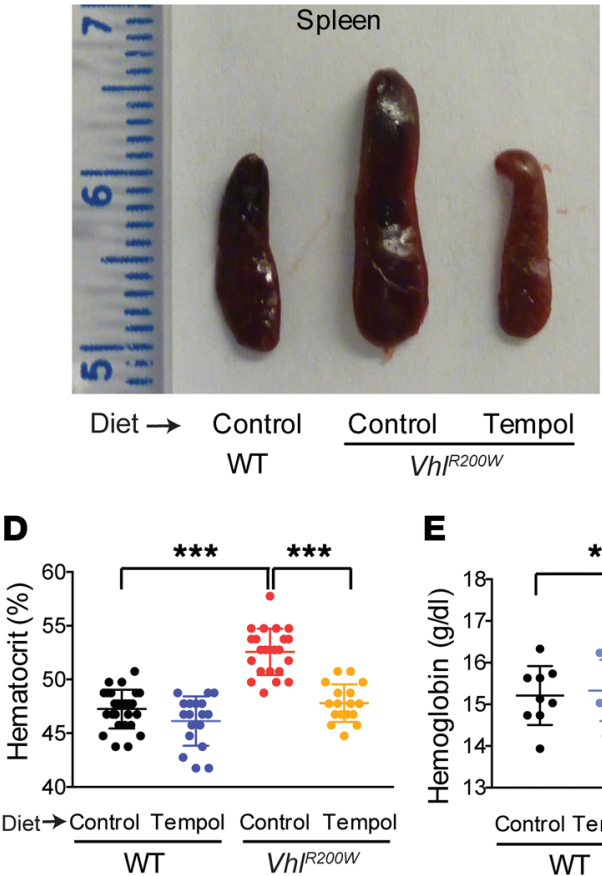

C

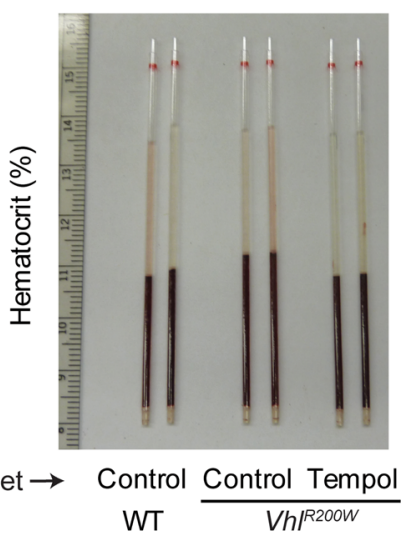

$\mathbf{F}$

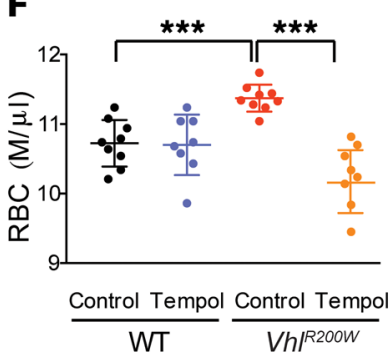

Figure 2. Dietary Tempol supplementation prevented plethora, splenomegaly, and erythrocytosis/polycythemia in Vh/ $^{\text {R200w }}$ mice. (A) The snouts and paws (indicated by white arrows) of $V h l^{R 200 w}$ mice appeared more reddish in color than did those of their WT littermates. The redness disappeared when the mutant mice were fed a Tempol-supplemented diet for 3 months. (B) Splenomegaly of $V h R^{\text {R20ow }}$ mice was rescued by dietary Tempol supplementation, and the color of the spleens became lighter, suggesting that Tempol supplementation ameliorated the extramedullary erythropoiesis. (C and D) Hematocrit levels, as determined by capillary tube centrifugation, and (E) hemoglobin and (F) RBC levels of 5- to 8-month-old WT and Vh/R20ow mice fed a control or Tempol-supplemented diet showed that with the control diet, these parameters were elevated in $V h^{\text {Rzoow }}$ mice, and Tempol supplementation significantly reduced hematocrit, hemoglobin, and RBC levels in the mutant mice. $(\mathbf{D}-\mathbf{F}){ }^{* * *} P<0.001$, by ordinary 1 -way ANOVA (multiple comparisons). tive to WT mice was not statistically significant. We measured serum EPO levels by the ELISA method and observed a small but significant increase in EPO in the sera of these mutant mice (Figure 3A), analogous to what was observed in Chuvash patients. Elevated serum EPO levels were significantly reduced when the mutant mice were treated with Tempol, indicating that Tempol corrected erythrocytosis/polycythemia in $V h l^{R 200 W}$ mice through downregulation of EPO.

Since EPO is a known specific transcriptional target of Hif $2 \alpha$ $(32,33)$, we assessed Hif $2 \alpha$ protein levels in nuclear fractions from renal lysates from WT mice on a control diet and $V h l^{R 200 W}$ mice maintained on either a control diet or a Tempol-supplemented diet, after harvesting intact kidneys in a nitrogen chamber and lysing the tissues in an anaerobic chamber. We found that Hif $2 \alpha$ protein levels were increased in $V h l^{R 200 W}$ mouse kidney lysates but significantly reduced when the mutant mice were fed the Tempol-supplemented diet (Figure 3, B and C). We measured the mRNA levels of other HIF targets. Although Hickey et al. (14) found modest but significantly higher expression of the Glut1 gene in $V h l^{R 200 W}$ kidney lysates, Glut 1 mRNA levels remained unchanged in the kidneys of our $V h l^{R 200 W}$ mice, nor did Tempol treatment alter the expression of Glut1 (Supplemental Figure 3A). Lung VEGF expression levels did not change in $V h l^{R 200 W}$ mice (Supplemental Figure 3B), as was the case with initial observations (34).
Downregulation of Hif $2 \alpha$ in Tempol-fed Vhl ${ }^{R 200 W}$ mice results from increased Hif $2 \alpha$ IRE-binding activity of Irp1. Hif $2 \alpha$ has an IRE in its 5'-UTR to which Irps can bind. To determine whether Tempol increased the Hif $2 \alpha$ IRE-binding activity of Irp 1 in these $V h l^{R 200 W}$ mice as previously observed in mouse embryonic fibroblast cells as well as in brain lysates from Irp2 $2^{-/-}$animals (29), we performed an RNA mobility shift assay with kidney lysates from WT and $V h l^{R 200 W}$ mice using radiolabeled ferritin IRE and Hif $2 \alpha$ IRE probes. Irp1 binding to both ferritin IRE and Hif $2 \alpha$ IRE probes markedly increased in Tempol-supplemented mice (Figure 3, $\mathrm{D}-\mathrm{F}$ ), though the Irp1 protein levels remained unchanged (Figure $3 G)$, indicating that increased binding of Irp1 with HIF $2 \alpha$ IRE in its 5'-UTR in Tempol-treated $V h l^{R 200 W}$ mice contributed substantially to translational repression of HIF $2 \alpha$.

To confirm that Tempol ameliorated erythrocytosis/polycythemia in $V h l^{R 200 W}$ mice through activation of Irp1 binding to the Hif $2 \alpha$ IRE, we fed Irp1 $1^{-/-}$mice with either a control or Tempol-supplemented diet and then evaluated the complete blood counts (CBCs) in these mice. The hematocrit levels of $\operatorname{Irp1^{-/-}}$ animals increased by more than $10 \%$ compared with those of WT mice on a control diet, as previously observed (18), but these elevated hematocrit levels did not decrease when $\operatorname{Irp1}^{-1-}$ mice were fed a Tempol-supplemented diet (Figure 4A), demonstrating 
A
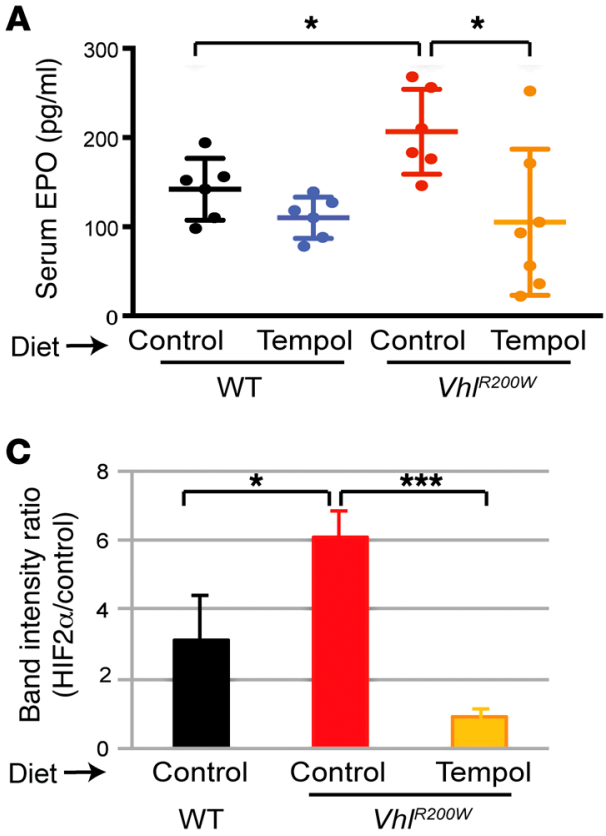

B

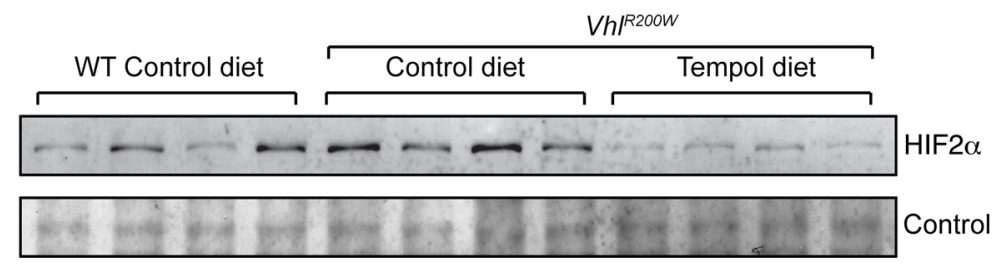

E

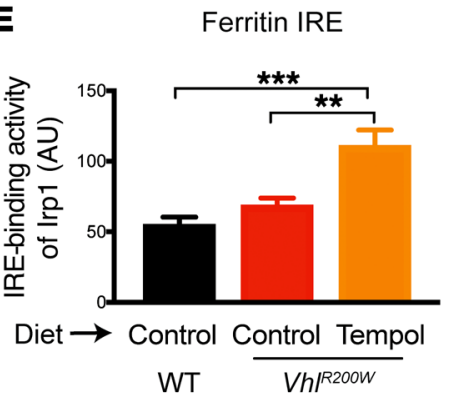

$\mathbf{F}$

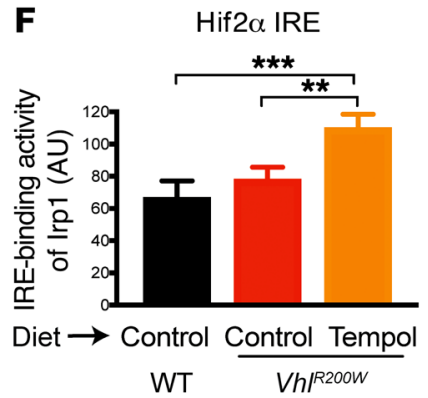

G $\quad W T$

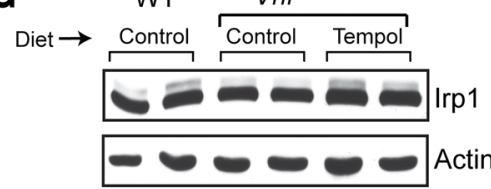

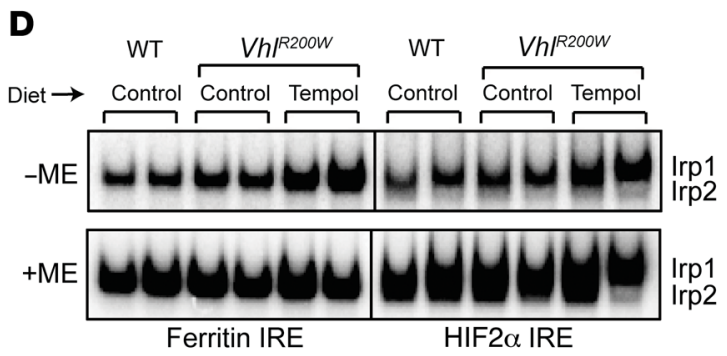

Ferritin IRE

HIF2 $\alpha$ IRE

Figure 3. Decreased erythropoiesis in Tempol-treated VhI $^{\text {R20ow }}$ mice was the result of EPO downregulation caused by translational repression of HIF2 $\alpha$, which was a consequence of the increased HIF2 $\alpha$ IRE-binding activity of Irp1. (A) Serum EPO levels measured by ELISA were significantly increased in control diet-fed Vh/R2oow mice, and Tempol treatment reduced EPO levels in these mutant mice. (B) Immunoblot analyses of nuclear fractions from kidney lysates showed increased HIF2 $\alpha$ protein levels in VhIR20ow mice fed a control diet, and the HIF2 $\alpha$ protein levels decreased when these mutant mice were fed a Tempol-supplemented diet. (C) Quantification of these HIF2 $\alpha$ protein levels. (D) RNA mobility shift assays for ferritin IRE and HIF2 $\alpha$ IRE binding of renal lysates in the absence or presence of $\beta$ mercaptoethanol (ME) (which converts Irp1 from cytosolic aconitase to the IRE-binding form) showed that most of the IRE-binding activity was contributed by Irp1, and Tempol treatment significantly increased the IRE binding of Irp1 in VhRR R2oow mice. Quantifications of the Irp1-binding activities of (E) ferritin IRE and (F) HIF2 $\alpha$ IRE. (G) Western blot analyses of kidney lysates revealed that Irp1 protein levels remained unchanged in Tempol diet-fed Vh/R20ow mice. Data in $\mathbf{A}, \mathbf{C}, \mathbf{E}$, and $\mathbf{F}$ represent the mean \pm SD. ${ }^{*} P \leq 0.05,{ }^{* *} P \leq 0.01$, and ${ }^{* *} P<0.001$, by unpaired, 2 -tailed $t$ test.

that Tempol did not lead to the suppression of hematocrit levels in the absence of Irp1. Moreover, we crossed $V h l^{R 200 W}$ heterozygous $\left(V h l^{R /+}\right)$ mice with $I r p 1^{-/-}$animals and bred the $V h l^{R /+} I r p^{+/-}$ mice to generate $V h l^{R 200 W} \operatorname{Irp1}^{-/-}$mice. We maintained these mice on either a control or Tempol-supplemented diet after weaning. Strikingly, hematocrit levels were elevated to as high as $84 \%$ in these $V h l^{R 200 W} \mathrm{Irp1}^{-/-}$mice, indicating that impaired degradation of Hif $2 \alpha$ caused by the homozygous $R 200 \mathrm{~W}$ mutations in the Vhl gene, combined with translational derepression of Hif $2 \alpha$ caused by the targeted deletion of Irp1, led to markedly increased erythropoiesis in these mice. These highly elevated hematocrit values did not significantly decrease upon Tempol treatment (Figure 4A), further confirming that Tempol attenuates erythrocytosis/ polycythemia through Irp1 activation and demonstrating that deletion of Irp 1 in mice blocks the beneficial effects of Tempol.
$\mathrm{RBC}$ and hemoglobin levels were also very high in $\mathrm{Vhl} \mathrm{l}^{\mathrm{R} 200 \mathrm{~W}} \mathrm{Irp1}^{\mathrm{T}^{-1}}$ mice, and these blood parameters were also not significantly decreased with Tempol treatment (Figure 4, B and C). In addition, Tempol did not significantly alter the RBC indices or reticulocyte, WBC, or platelet counts of $\operatorname{Irp1}^{-/-}$or $V h l^{R 200 W} \mathrm{Irp1}^{-/-}$mice. (Supplemental Figure 4, A-F). EPO levels of the control diet-fed $V h l^{R 200 W} \mathrm{Irp1}^{-/ /}$mice were 3.3-fold higher than those of WT mice maintained on the control diet (Figure 4D), whereas we detected a significant 1.5-fold increase of EPO levels in $V h l^{R 200 W}$ mice, which indicated that Irp1 is a physiologic regulator of Hif $2 \alpha$ and erythropoietin expression levels.

Tempol represses hypoxia-induced erythrocytosis/polycythemia in WT mice. Humans living at high altitudes face survival challenges because of erythrocytosis/polycythemia and ensuing complications caused by hypobaric hypoxia $(35,36)$. To determine whether Tempol 
A

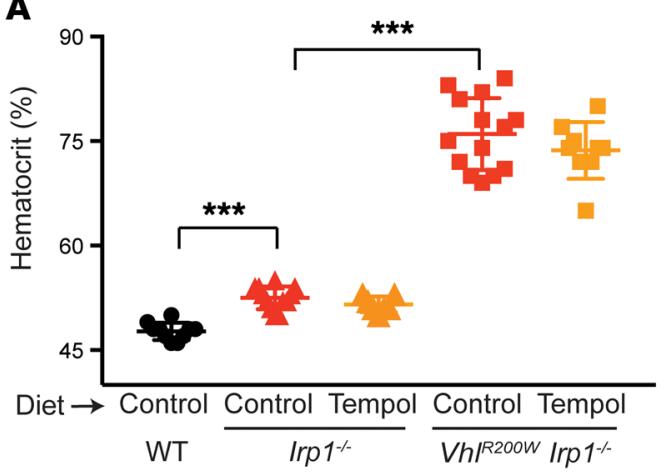

C

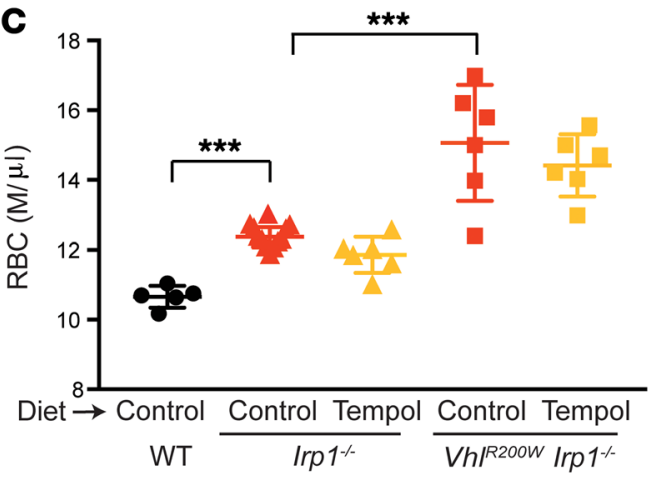

B

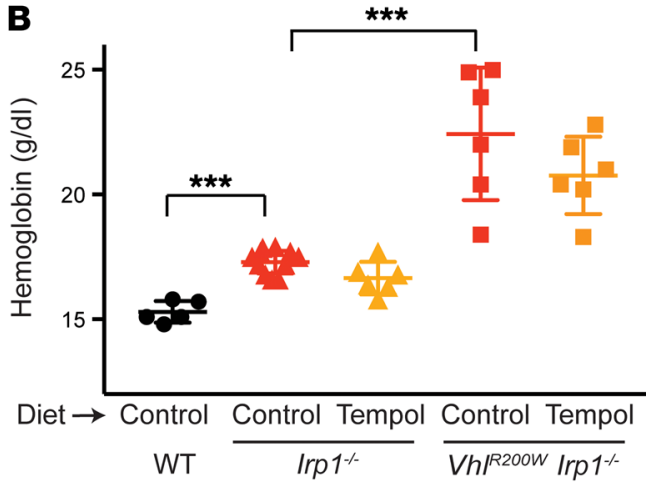

D

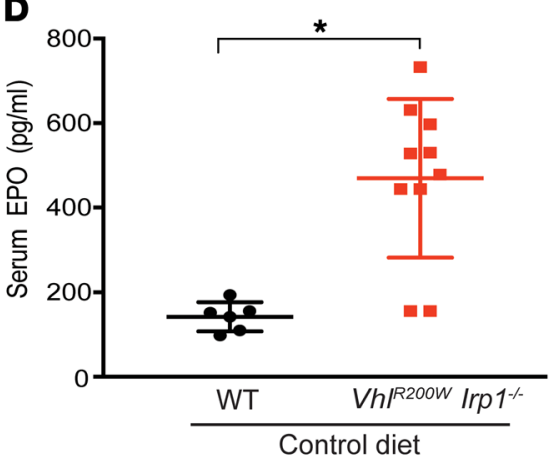

E

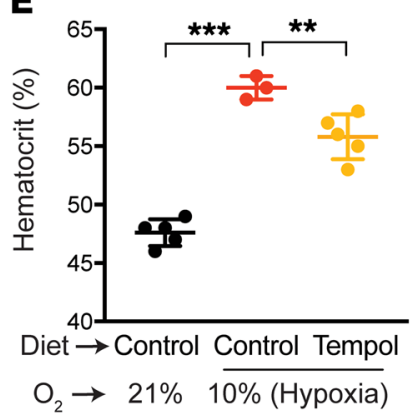

F

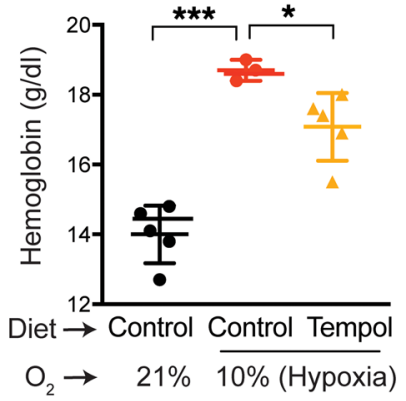

G

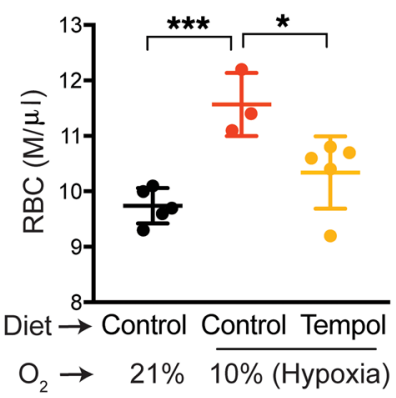

Figure 4. Genetic ablation of Irp1 significantly increased polycythemia inVh/200w mice, which became refractory to Tempol, confirming that Tempol activates Irp1 to ameliorate the polycythemia in VhR ${ }^{200 w}$ mice, and Tempol also partially protects WT mice from hypoxic polycythemia/erythrocytosis. (A) Hematocrit (determined by capillary tube centrifugation), (B) hemoglobin, and (C) RBC levels of 5- to 8-month-old WT, Irp1 $1^{-1-}$, and Vh/R20ow $/ \mathrm{rp} 1^{1 /-}$ mice fed a control or Tempol-supplemented diet revealed that Tempol did not decrease erythropoiesis when Irp1 was ablated in the mice. (D) Serum EPO levels of control diet-fed Vh/R200W $/ \mathrm{rpt}^{-1-}$ mice were 3.3-fold higher than were those of WT mice (data for WT mice are the same as those in Figure 3A). (E) Hematocrit, (F) hemoglobin, and (G) RBC levels of 10-month-old mice on a control diet or Tempol diet after placing them in normoxic or hypoxic (10\% oxygen) conditions showed that blood parameters significantly increased in the hypoxic control diet-fed mice and that Tempol supplementation partially prevented erythrocytosis/polycythemia in these mice. ${ }^{*} P \leq 0.05,{ }^{* *} P \leq 0.01$, and ${ }^{* *} P<0.001$, by 1-way ANOVA.

could attenuate erythrocytosis/polycythemia in mice that developed erythrocytosis/polycythemia caused by hypoxia, we placed WT mice fed a control or Tempol-supplemented diet into a hypoxia chamber containing $10 \%$ oxygen for 23 days and then measured RBC parameters. We found that the hematocrit, hemoglobin, and RBC levels of these mice increased significantly under hypoxic conditions (Figure 4, E-G). Tempol diet feeding significantly reduced the levels of hematocrit, hemoglobin, and RBC levels in hypoxic mice compared with control diet-fed mice (Figure 4, E-G), indicating that Tempol partially protected these hypoxic WT mice from developing erythrocytosis/polycythemia. Importantly, 2 of the 5 mice fed a control diet died while they were in the hypoxic chamber, whereas all 5 Tem- pol-fed hypoxic mice survived, suggesting that Tempol prevented mortality in mice exposed to hypoxic environments.

Tempol increases the survival of $V h l^{R 200 W}$ mice. We studied 1-year survival rates of WT, $V h l^{R 200 W}$, and $V h l^{R 200 W} \mathrm{Irp1}^{-/-}$mice maintained on a control or Tempol-supplemented diet and observed that the mortality rates of $V h l^{R 200 W}$ mice were significantly higher than those of WT mice and that Tempol supplementation changed the survival rates of $V h l^{R 200 W}$ mice from $87 \%$ to $93 \%$ (Figure 5), again suggesting a beneficial effect of Tempol on these mutant mice. However, survival rates did not increase with Tempol supplementation when Irp1 was deleted from these $V h l^{R 200 W}$ mice, reconfirming that Tempol acts primarily through Irp1 activation (Figure 5). 


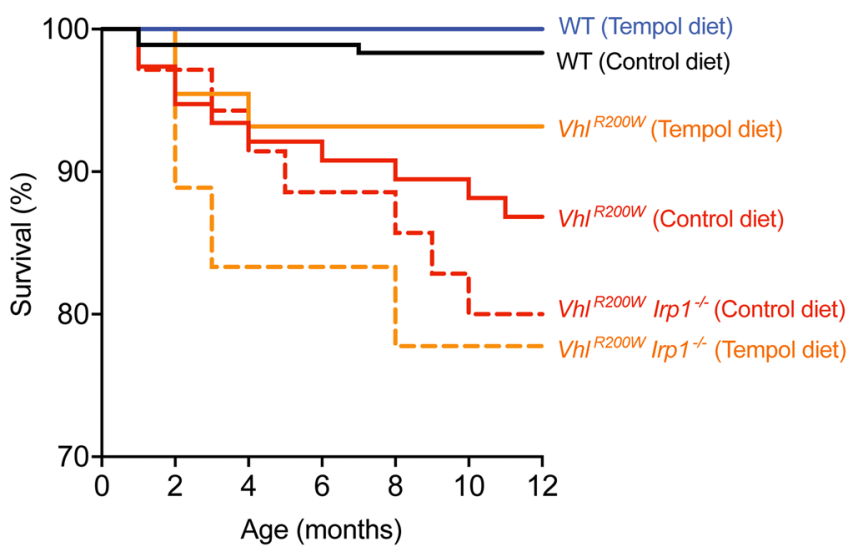

\section{Discussion}

In this study, we showed that activation of Irp1 in $V h l^{R 200 w}$ mice by dietary Tempol supplementation repressed Hif $2 \alpha$ expression, decreased EPO production, ameliorated erythrocytosis/polycythemia, and increased the lifespans of these mice (Figure 6). Until recently, phlebotomy therapy had been the only known treatment for patients with Chuvash polycythemia, but it is not clear whether phlebotomy actually attenuates complications or reduces the mortality of these patients (13). On the contrary, frequent phlebotomies cause iron deficiency that can lead to two opposing effects on HIF $2 \alpha$ expression. Iron deficiency exerts a direct stabilizing effect on HIF $2 \alpha$ by inhibiting PHD2 activity and subsequently reducing its proteasomal degradation, whereas increased binding of IRP1 to the HIF $2 \alpha$ IRE at low iron concentrations leads to translational repression of HIF2 $\alpha$. Overall, phlebotomies may increase HIF $2 \alpha$ expression, induce iron deficiency in essential nonerythropoietic tissues, and fail to sufficiently repress erythrocytosis/polycythemia. It is important to note here that both HIF1 $\alpha$ and HIF $2 \alpha$ are expected to increase in many iron deficiency conditions, but the IRE present in the HIF2 $\alpha$ transcript, but not in the HIF1 $\alpha$ transcript, dictates that its translation will be repressed in iron-depleted cells that are rich in IRP1 $(16,18)$.

HIF2 $\alpha$ is also associated with many other VHL diseases including CCRCCs, and targeting HIF2 $\alpha$ is of tremendous interest for the treatment of patients with such diseases $(37,38)$. Small-molecule Hif2 $\alpha$ inhibitors have been found to improve VHL diseaseassociated phenotypes in zebrafish (39) and in mouse models (37, 40 ), and some of these inhibitors are now in clinical trials. Thus, Tempol therapy can be very useful to treat the diseases associated with upregulation of HIF $2 \alpha$ expression or mutation in the $V H L$ or PHD2 gene. Tempol was also previously found to protect Irp2 $2^{--}$mice from neurodegeneration through disassembly of the iron-sulfur cluster of Irp1, activating its IRE-binding activity and thus partially compensating for the loss of Irp2 in those cells and tissues in which Irp2 was the main functional Irp (29).

Tibetans and people living in high-altitude regions for generations have developed genetic adaptations and shown improved survival, despite living with decreased oxygen availability $(41,42)$. A high-frequency missense mutation in the EGLN1 gene encoding PHD2 was recently found to be responsible for the adaptive response in Tibetans (43). However, for most of the nonadapted
Figure 5. The reduced life expectancy of $\mathbf{V h}^{\text {R200w }}$ mice was alleviated by Tempol supplementation when Irp1 was present. The survival curves of WT, $V h h^{R 200 W}$, and $V h h^{R 200 W} /$ rpt $^{1-1-}$ mice on a control or Tempol-supplemented diet showed that $V h l^{R 200 w}$ mice on a control diet died at significantly higher survival rates than did WT mice on a control diet, whereas no significant difference was observed in the survival rates between Tempol-supplemented $V h h^{R 200 W}$ and WT mice (on either a control or Tempol-supplemented diet). Moreover, the beneficial effect of Tempol was lost when Irp1 was absent in these Vhl ${ }^{R 200 W}$ mice. $n=180$ WT mice on a control diet; $n=34$ WT mice on a Tempol diet; $n=76 \mathrm{Vh} \mathrm{h}^{\text {R200W }}$ mice on a control diet; $n=43 \mathrm{Vh} \mathrm{R}^{\text {R200W }}$ mice on a Tempol diet; $n=35 \mathrm{Vh}^{R 200 \mathrm{~W}} / \mathrm{Irp1}^{-1 /-}$ mice on a control diet; and $n=18 \mathrm{Vh}^{\text {R20ow }} / \mathrm{rpt}^{-/-}$mice on a Tempol diet . Statistical analyses of mouse survival curves were performed with a log-rank Mantel-Cox test using GraphPad Prism software.

people, exposure to high-altitude hypoxia causes erythrocytosis/ polycythemia characterized by increased erythropoiesis and elevated hematocrit levels. Our results showed that Tempol therapy ameliorates hypoxic erythrocytosis/polycythemia, analogous to human hypoxia, indicating that Tempol could constitute a therapeutic approach to treat high-altitude erythrocytosis/polycythemia in nonadapted humans.

Our results showed hypersensitivity to EPO, which is a hallmark of Chuvash polycythemia patients $(44,45)$ and the Chuvash polycythemia $V h l^{R 200 W}$ mouse model (14). In normal individuals, VHL binds to the suppressor of cytokine signaling 1 (SOCS1) protein and promotes ubiquitin-mediated degradation of phosphorylated Janus kinase 2 (p-JAK2), thus blocking the JAK2/STAT5 pathway (46). In $V h l^{R 200 w}$ mice, mutated VHL was reported to bind to SOCS1 more tightly, thus reducing the degradation of p-JAK2 (46). As a result, stabilized p-JAK2 was proposed to hyperactivate the JAK2/STAT5 pathway, leading to EPO hypersensitivity in erythroid progenitors (46). Treatment of 3 Chuvash polycythemia patients with ruxolitinib, an inhibitor of JAK1 and JAK2, was recently shown to reduce the symptoms of erythrocytosis/polycythemia and the need for frequent phlebotomy treatments (47). However, our results suggest that increased HIF2 $\alpha$ expression is the more important factor in causing erythrocytosis/polycythemia in $V h l^{R 200 W}$ mice.

Our present study shows that Irp1 functions as a critical regulator of erythropoietin expression through its ability to repress Hif $2 \alpha$ translation when it is in its apo form, which lacks a Fe-S cluster, by binding to the Hif $2 \alpha$ IRE. We have discovered that the use of Tempol, a stable nitroxide known to disassemble the Fe-S cluster in Irp1 (29), leads to translational repression of Hif2 $\alpha$ in a Chuvash polycythemia mouse model. Other drug therapy approaches have involved interfering with the functions of HIF $2 \alpha$ itself or with downstream sensitivities to HIF $2 \alpha$. Our proposed treatment involves the repression of HIF $2 \alpha$ translation, and it could be of great therapeutic value alone or in conjunction with other proposed treatments, since it acts upstream of other therapies. Since manipulations of iron status cause competing effects on HIF $2 \alpha$ expression, we propose that targeting the upstream translational regulator IRP1 may offer a more straightforward treatment and better outcomes in patients whose prognosis remains relatively unimproved by current treatment strategies. Our proposed approach is also feasible and potentially cost effective. In summary, the results of our study, which demonstrate an important role for translational regulation of 

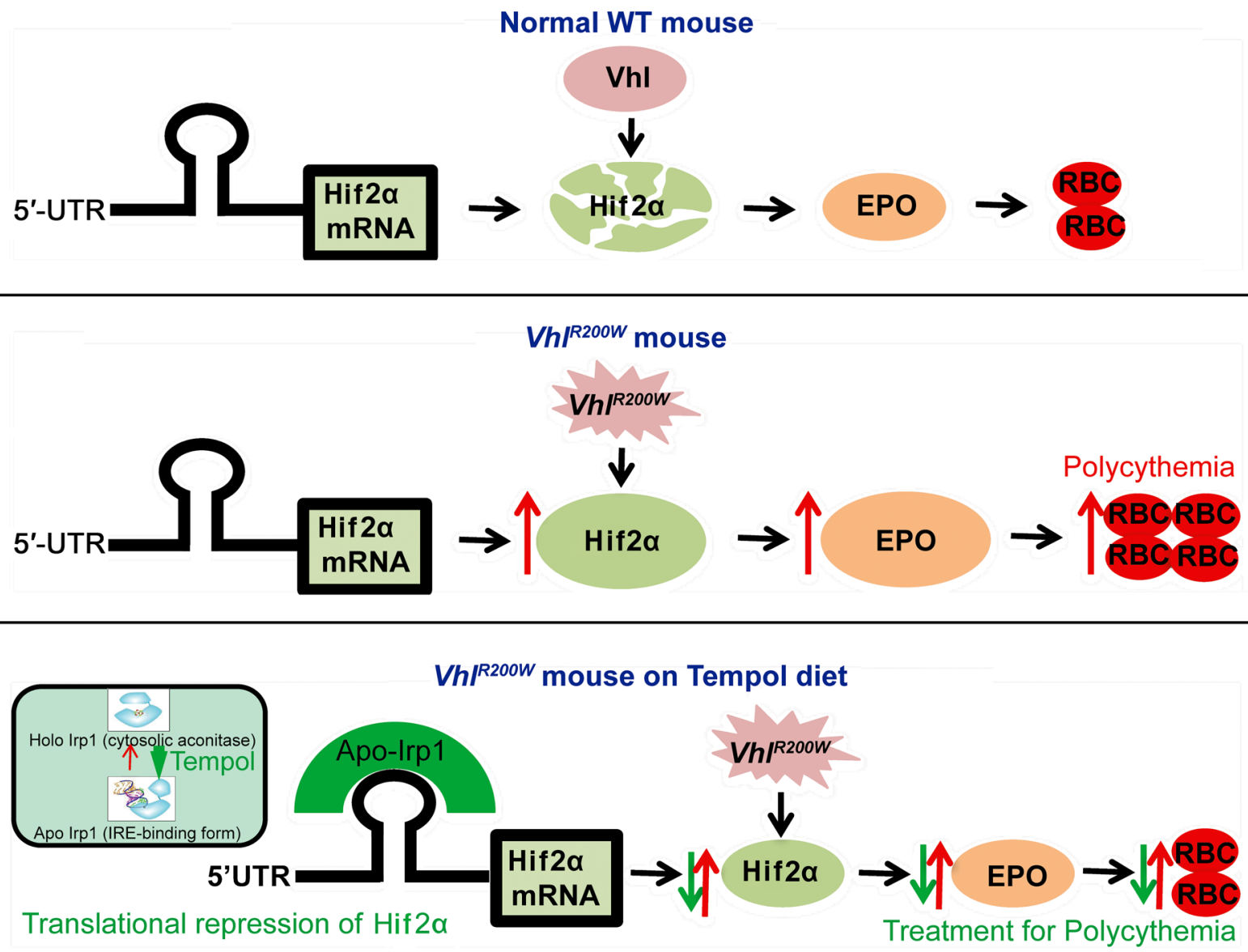

Figure 6. Model for the mechanism of ameliorative action of Tempol in Vh/R20ow mice. The expression of HIF2 $\alpha$ protein is regulated at multiple levels. The $\mathrm{Vhl}$ protein promotes the degradation of HIF2 $\alpha$ under normoxic conditions. However, Vh/R20ow does not promote HIF2 $\alpha$ degradation, which leads to high levels of HIF2 $\alpha$ and augmented levels of translational targets, including EPO. HIF2 $\alpha$ is also translationally regulated by the Irp IRE regulatory system. When Tempol increases the IRE-binding activity of Irp1, HIF2 $\alpha$ expression diminishes, which leads to diminished erythropoietin expression and restoration of normal hematocrit levels.

HIF $2 \alpha$ in these disease settings, suggest that oral Tempol administration should be explored as a therapeutic intervention to prevent erythrocytosis/polycythemia in patients with Chuvash polycythemia and in nonadapted individuals living at high altitude who suffer from erythrocytosis/polycythemia.

\section{Methods}

Study design. On the basis of our previous findings $(18,29)$, we hypothesized that Tempol would activate the IRE-binding activity of Irp1, which would thereby inhibit Hif $2 \alpha$ translation and diminish polycythemia in $V h l^{R 200 W}$ mice. We measured hematocrit levels, which are the hallmark for erythrocytosis/polycythemia, using the capillary spin method. We also used an IDEXX hematoanalyzer to obtain hematocrit values for WT and Vhl ${ }^{R 200 W}$ mice on a control or Tempol-supplemented diet. Next, we used ELISA assays to determine serum EPO levels, since increased EPO production was found to cause erythrocytosis/ polycythemia in $V h l^{R 200 W}$ polycythemia patients. Next, we assessed Hif $2 \alpha$ levels by Western blotting, because EPO is a target of Hif $2 \alpha$. Since HIF $2 \alpha$ has an IRE in its $5^{\prime}$-UTR, we assessed whether the Hif $2 \alpha$ IRE-binding activity of Irp1 was increased in Tempol-supplemented $V h l^{\text {R20ow }}$ mice by performing RNA mobility shift assays, also known as RNA gel shift or RNA bandshift assays, after incubating the renal cell lysate with $\mathrm{P}^{32}$-labeled Hif2 $\alpha$ IRE. We did not exclude any data. In all experiments, animals were randomized to different treatment groups. For the survival study experiment (Figure 5), sample sizes are specified in the figure legend, and for all other experiments, sample sizes are indicated in the figures. For Supplemental Figures 2-4, the sample sizes are specified in each figure legend.

Animals. The $V h l^{\text {R20oW }}$ mice were originally generated by Hickey et al. (14). Heterozygous breeding pairs (C57BL/6 background) (48) were provided by Mary Slingo and Peter A. Robbins (University of Oxford, Oxford, United Kingdom). The mice were weaned at 3 to 4 weeks of age, genotyped as described by Hickey et al., and maintained on a normal NIH-07 diet unless otherwise noted. For diet experiments, WT and $V h l^{\text {R20ow }}$ littermates were placed on a control diet or on the same diet supplemented with Tempol at 6 to 8 weeks of age and were maintained on these diets until used for experiments. $\operatorname{Irp1^{-/}}$ mice were generated as previously described $(49,50)$ and backcrossed with C57BL/6 mice for 10 generations. $V h l^{R 200 W I r p 1-/-}$ mice were generated by first crossing $V h l^{R 200 W}$ heterozygous $\left(V h l^{R /+}\right)$ mice with $I r p 1^{-/-}$mice, and then breeding the $V h l^{R / I r p+/-}$ mice. All the experiments were performed with 5- to 8-month-old mice unless noted otherwise. Mice of different genotypes and on different diets were age matched. 
Diet description. The Tempol diet was prepared by uniformly mixing powdered Tempol (Sigma-Aldrich) with bacon-flavored mouse chow (Bio-Serv) by a cold-press technique at a concentration of $10 \mathrm{mg} / \mathrm{g}$ food. Bacon-flavored chow without Tempol was used as the control diet (29).

Hematocrit measurement (capillary spin method). Approximately $100 \mu \mathrm{l}$ blood was harvested from each mouse by mandibular bleeding (from the facial vein) into a heparin-coated BD microtainer tube with lithium heparin. Hematocrit tubes were $80 \%$ filled with blood by capillary action, sealed, and centrifuged in a microhematocrit centrifuge (LW Scientific). Hematocrit values were measured with a hematocrit reader. Unless otherwise noted, all the hematocrit data presented were measured using this capillary spin method.

$C B C$ measurement. Approximately $100 \mu$ blood was harvested from each mouse by mandibular bleeding and placed into a heparin-coated tube. Hemoglobin and RBC levels of mice for the hypoxia experiment (Figure 4, F and G) were obtained from the CBC measurement on a Hemavet 1500 Analyzer (Drew Scientific). All other CBC measurements were done with an IDEXX ProCyte Dx Hematology Analyzer.

Serum erythropoietin and serum ferritin measurements by ELISA. Erythropoietin and ferritin were measured in serum samples with ELISA kits from R\&D Systems and Abcam, respectively, according to the manufacturers' protocols.

Tissue and lysate preparation. Animals were euthanized using $\mathrm{CO}_{2}$, and the tissues were harvested immediately inside a nitrogen chamber. Fresh tissues were lysed inside the anaerobic chamber in lysis buffer that was de-aerated by cyclic freeze-thawing and air removal with argon to stabilize Hif $2 \alpha$.

RNA mobility shift assay. The RNA mobility shift assay was performed as previously described (49). Tissue lysates were prepared in an anaerobic chamber, as described in the Tissue and lysate preparation section, in oxygen-depleted lysis buffer containing $10 \mathrm{mM}$ HEPES (pH 7.2), $3 \mathrm{mM} \mathrm{MgCl} 2,40 \mathrm{mM} \mathrm{KCl}, 5 \%$ glycerol, $0.2 \%$ nonidet P- 40 , $5 \mathrm{mM}$ DTT, $1 \mathrm{mM}$ AEBSF, $10 \mu \mathrm{g} / \mathrm{ml}$ leupeptin, and Complete EDTAFree Protease Inhibitor Mixture (Roche Applied Science). Lysate was added at a volume containing $10 \mu \mathrm{g}$ total protein to bandshift buffer containing $25 \mathrm{mM}$ Tris- $\mathrm{HCl}$ (pH 7.5) and $40 \mathrm{mM} \mathrm{KCl}$, with or without $2 \% \beta$-mercaptoethanol, a chemical that activates Irp1 in vitro. The resulting sample solutions were incubated for 5 minutes at room temperature with $12.5 \mu \mathrm{l}$ reaction mixture containing $20 \%$ glycerol, 0.2 units/ $\mu$ l Super RNAsine, $0.6 \mu \mathrm{g} / \mu \mathrm{l}$ yeast transfer RNA (tRNA), $5 \mathrm{mM}$ DTT, and $20 \mathrm{nM}{ }^{32} \mathrm{P}$-labeled IRE from the human ferritin $\mathrm{H}$ chain gene (51) or $60 \mathrm{nM}{ }^{32} \mathrm{P}$-labeled IRE from the HIF2 $\alpha$ gene (52) in $25 \mathrm{mM}$ Tris- $\mathrm{HCl}(\mathrm{pH} 7.5)$ and $40 \mathrm{mM} \mathrm{KCl}$. A 20- $\mu$ l amount of the resulting reaction mixture was loaded into $10 \%$ acrylamide/TBE gel, which was run at $200 \mathrm{~V}$ for 2.15 hours, and the gel was fixed, dried, and exposed for autoradiography. Quantitation was performed with the ImageQuant program for Macintosh (GE Healthcare)

Western blot analysis and antibodies. Tissue lysates were prepared in an anaerobic chamber in oxygen-depleted lysis buffer containing $50 \mathrm{mM}$ Tris- $\mathrm{HCl}$ (pH 7.5), $150 \mathrm{mM} \mathrm{NaCl}, 0.1 \%$ SDS, $0.5 \%$ sodium deoxycholate, $1 \%$ Triton X-100, 1 mM PMSF, and Complete EDTA-Free Protease Inhibitor Mixture. Protein analysis with the anaerobic lysates was carried out as previously described (53). The lysates were added to sample buffers inside an anaerobic chamber for Western blot experiments. Equal amounts of protein (20-50 $\mu \mathrm{g}$ /lane) were separated on 10\% SDS-PAGE gels and transferred onto nitrocellulose membranes. The membrane was blocked with 5\% nonfat milk and 0.1\% Triton X-100 in PBS and probed at room temperature in the same blocking buffer. IRP1 antibody was prepared against purified human IRP1 and used at a 1:5,000 dilution. Hif2 $\alpha$ Western blotting was done with nuclear fractions from kidney lysates after harvesting intact kidneys in a nitrogen chamber and lysing the tissues in an anaerobic chamber. Hif $2 \alpha$ antibody (affinity-purified goat anti-human) was obtained from R\&D Systems (catalog AF2886), and a final concentration of $1.0 \mu \mathrm{g} / \mathrm{ml}$ was used as described in the R\&D Systems protocol. Monoclonal anti- $\beta$-actin antibody (Sigma-Aldrich) was used at a 1:5,000 dilution. Western blots were treated with secondary peroxidase-conjugated goat anti-rabbit IgG or sheep anti-mouse IgG antibodies (GE Healthcare) at 1:5,000 and 1:2,000 dilutions, respectively.

Statistics. Data are expressed as the mean \pm SD. Comparisons between 2 groups were analyzed using an unpaired, 2-tailed $t$ test. Analyses of multiple groups were performed using an ordinary 1-way ANOVA (multiple comparisons). $P$ values of less than 0.05 were considered statistically significant. Statistical analyses of mouse survival curves were performed with a log-rank (Mantel-Cox) test (curve comparison) using GraphPad Prism 7.0 (GraphPad Software).

Study approval. All procedures involving mice were performed following protocols approved by the IACUC of the NICHD (protocol 15-038) and met NIH guidelines for the humane care of animals.

\section{Author contributions}

MCG designed the project, performed most of the experiments, analyzed the data, and wrote the manuscript. DLZ performed experiments, analyzed data, and reviewed the manuscript. HO performed experiments and reviewed the manuscript. MAE performed experiments and reviewed the manuscript. TAR designed and supervised the project and wrote the manuscript.

\section{Acknowledgments}

This work was supported by the Eunice Kennedy Shriver NICHD Intramural Research Program. We thank Peter Robbins and Mary Slingo (University of Oxford) for providing the heterozygous $V h l^{R 200 W}$ mice; Dan Crooks of the National Cancer Institute (NCI) for making cold HIF $2 \alpha$ IRE; Jorge Chavez of the Division of Veterinary Resources for performing some of the $\mathrm{CBC}$ and serum chemistry measurements; Michele D. Allen of the National Heart, Lung, and Blood Institute (NHLBI) for helping with the mouse hypoxia chamber; James B. Mitchell of the NCI for his intellectual input and for reading the manuscript; Anastasia Showers of the NCI for helping prepare the Tempol diet food; and Marston Linehan of the NCI and the Rouault laboratory members for contributing to constructive discussions.

Address correspondence to: Tracey A. Rouault, 35A Convent Drive, Room 2D824, National Institutes of Health, Bethesda, Maryland 20892, USA. Phone: 301.496.7060; Email: rouault@mail.nih.gov.
1. Poliakova LA. [Familial erythrocytosis among the residents of the Chuvash ASSR]. Probl Gematol Pereliv Krovi. 1974;19(10):30-33.

2. Miasnikova GY, et al. The heterozygote advan- tage of the Chuvash polycythemia VHLR200W mutation may be protection against anemia. Haematologica. 2011;96(9):1371-1374.

3. Sergeyeva A, Gordeuk VR, Tokarev YN, Sokol L,
Prchal JF, Prchal JT. Congenital polycythemia in Chuvashia. Blood. 1997;89(6):2148-2154.

4. Krek W. VHL takes HIF's breath away. Nat Cell Biol. 2000;2(7):E121-E123. 
5. Tyers M, Rottapel R. VHL: a very hip ligase. Proc Natl Acad Sci U S A. 1999;96(22):12230-12232.

6. Lanikova L, et al. Novel homozygous VHL mutation in exon 2 is associated with congenital polycythemia but not with cancer. Blood . 2013;121(19):3918-3924.

7. Tomasic NL, et al. The phenotype of polycythemia due to Croatian homozygous VHL (571C > G:H191D) mutation is different from that of Chuvash polycythemia (VHL 598C >T:R200W). Haematologica. 2013;98(4):560-567.

8. Pastore YD, et al. Mutations in the VHL gene in sporadic apparently congenital polycythemia. Blood.2003;101(4):1591-1595.

9. Nordstrom-O'Brien M, et al. Genetic analysis of von Hippel-Lindau disease. Hum Mutat. 2010;31(5):521-537.

10. Haase VH. The VHL/HIF oxygen-sensing pathway and its relevance to kidney disease. Kidney Int. 2006;69(8):1302-1307.

11. Rankin EB, et al. Hypoxia-inducible factor-2 (HIF-2) regulates hepatic erythropoietin in vivo. JClin Invest. 2007;117(4):1068-1077.

12. Gordeuk VR, et al. Congenital disorder of oxygen sensing: association of the homozygous Chuvash polycythemia VHL mutation with thrombosis and vascular abnormalities but not tumors. Blood. 2004;103(10):3924-3932.

13. Gordeuk VR. Chuvash polycythemia: diagnosis and management. Clin Adv Hematol Oncol. 2011;9(12):929-930.

14. Hickey MM, Lam JC, Bezman NA, Rathmell WK, Simon MC. von Hippel-Lindau mutation in mice recapitulates Chuvash polycythemia via hypoxia-inducible factor-2 $\alpha$ signaling and splenic erythropoiesis. J Clin Invest. 2007;117(12):3879-3889.

15. Frise MC, et al. Clinical iron deficiency disturbs normal human responses to hypoxia. JClin Invest. 2016;126(6):2139-2150.

16. Rouault TA. The role of iron regulatory proteins in mammalian iron homeostasis and disease. Nat Chem Biol. 2006;2(8):406-414.

17. Zhang DL, Ghosh MC, Rouault TA. The physiological functions of iron regulatory proteins in iron homeostasis - an update. Front Pharmacol. 2014;5:124.

18. Ghosh MC, et al. Deletion of iron regulatory protein 1 causes polycythemia and pulmonary hypertension in mice through translational derepression of HIF2 $\alpha$. Cell Metab. 2013;17(2):271-281.

19. Anderson SA, et al. The IRP1-HIF-2 $\alpha$ axis coordinates iron and oxygen sensing with erythropoiesis and iron absorption. Cell Metab. 2013;17(2):282-290.

20. Wilkinson N, Pantopoulos K. IRP1 regulates erythropoiesis and systemic iron homeostasis by controlling HIF $2 \alpha$ mRNA translation. Blood. 2013;122(9):1658-1668.

21. Wilkinson N, Pantopoulos K. The IRP/IRE system in vivo: insights from mouse models. Front Pharmacol. 2014;5:176.
22. Mitchell JB, et al. Biologically active metal-independent superoxide dismutase mimics. Biochemistry. 1990;29(11):2802-2807.

23. Bonini MG, Mason RP, Augusto O. The Mechanism by which 4-hydroxy-2,2,6,6-tetramethylpiperidene-1-oxyl (tempol) diverts peroxynitrite decomposition from nitrating to nitrosating species. Chem Res Toxicol. 2002;15(4):506-511.

24. Mitchell JB, et al. A low molecular weight antioxidant decreases weight and lowers tumor incidence. Free Radic Biol Med. 2003;34(1):93-102.

25. Deng-Bryant Y, Singh IN, Carrico KM, Hall ED. Neuroprotective effects of tempol, a catalytic scavenger of peroxynitrite-derived free radicals, in a mouse traumatic brain injury model. J Cereb Blood Flow Metab. 2008;28(6):1114-1126.

26. Kato N, Yanaka K, Hyodo K, Homma K, Nagase $\mathrm{S}$, Nose T. Stable nitroxide Tempol ameliorates brain injury by inhibiting lipid peroxidation in a rat model of transient focal cerebral ischemia. Brain Res. 2003;979(1-2):188-193.

27. Lipman T, Tabakman R, Lazarovici P. Neuroprotective effects of the stable nitroxide compound Tempol on 1-methyl-4-phenylpyridinium ion-induced neurotoxicity in the Nerve Growth Factor-differentiated model of pheochromocytoma PC12 cells. Eur J Pharmacol. 2006;549(1-3):50-57.

28. Metz JM, et al. A phase I study of topical Tempol for the prevention of alopecia induced by whole brain radiotherapy. Clin Cancer Res. 2004;10(19):6411-6417.

29. Ghosh MC, et al. Tempol-mediated activation of latent iron regulatory protein activity prevents symptoms of neurodegenerative disease in IRP2 knockout mice. Proc Natl Acad Sci U S A. 2008;105(33):12028-12033.

30. Ghosh MC, Zhang DL, Rouault TA. Iron misregulation and neurodegenerative disease in mouse models that lack iron regulatory proteins. Neurobiol Dis. 2015;81:66-75.

31. Cohen LA, et al. Serum ferritin is derived primarily from macrophages through a nonclassical secretory pathway. Blood. 2010;116(9):1574-1584

32. Semenza GL. Hypoxia-inducible factors: mediators of cancer progression and targets for cancer therapy. Trends Pharmacol Sci. 2012;33(4):207-214.

33. Keith B, Johnson RS, Simon MC. HIF1 $\alpha$ and HIF2 $\alpha$ : sibling rivalry in hypoxic tumour growth and progression. Nat Rev Cancer. 2011;12(1):9-22.

34. Hickey MM, et al. The von Hippel-Lindau Chuvash mutation promotes pulmonary hypertension and fibrosis in mice. J Clin Invest. 2010;120(3):827-839.

35. Cheviron ZA, Brumfield RT. Genomic insights into adaptation to high-altitude environments. Heredity (Edinb). 2012;108(4):354-361.

36. Basang Z, et al. HIF2A variants were associated with different levels of high-altitude hypoxia among native Tibetans. PLoS One. 2015;10(9):e0137956.

37. Martínez-Sáez O, Gajate Borau P, Alonso-Gordoa
T, Molina-Cerrillo J, Grande E. Targeting HIF-2 $\alpha$ in clear cell renal cell carcinoma: A promising therapeutic strategy. Crit Rev Oncol Hematol. 2017;111:117-123.

38. Sourbier C, et al. Targeting HIF2 $\alpha$ translation with Tempol in VHL-deficient clear cell renal cell carcinoma. Oncotarget. 2012;3(11):1472-1482.

39. Metelo AM, et al. Pharmacological HIF2 $\alpha$ inhibition improves VHL disease-associated phenotypes in zebrafish model. J Clin Invest. 2015;125(5):1987-1997.

40. Chen $\mathrm{W}$, et al. Targeting renal cell carcinoma with a HIF-2 antagonist. Nature. 2016;539(7627):112-117.

41. Beall CM. Two routes to functional adaptation: Tibetan and Andean high-altitude natives. Proc Natl Acad Sci U S A. 2007;104(suppl 1):8655-8660.

42. Simonson TS, et al. Genetic evidence for high-altitude adaptation in Tibet. Science. 2010;329(5987):72-75.

43. Lorenzo FR, et al. A genetic mechanism for Tibetan high-altitude adaptation. Nat Genet. 2014;46(9):951-956.

44. Ang SO, et al. Endemic polycythemia in Russia: mutation in the VHL gene. Blood Cells Mol Dis. 2002;28(1):57-62.

45. Ang SO, et al. Disruption of oxygen homeostasis underlies congenital Chuvash polycythemia. Nat Genet. 2002;32(4):614-621.

46. Russell RC, et al. Loss of JAK2 regulation via a heterodimeric VHL-SOCS1 E3 ubiquitin ligase underlies Chuvash polycythemia. Nat Med. 2011;17(7):845-853.

47. Zhou AW, Knoche EM, Engle EK, Ban-Hoefen M, Kaiwar C, Oh ST. Clinical Improvement with JAK2 Inhibition in Chuvash Polycythemia. N Engl JMed. 2016;375(5):494-496.

48. Slingo M, et al. The von Hippel-Lindau Chuvash mutation in mice alters cardiac substrate and high-energy phosphate metabolism. Am J Physiol Heart Circ Physiol. 2016;311(3):H759-H767.

49. Meyron-Holtz EG, et al. Genetic ablations of iron regulatory proteins 1 and 2 reveal why iron regulatory protein 2 dominates iron homeostasis. EMBO J. 2004;23(2):386-395.

50. Meyron-Holtz EG, Ghosh MC, Rouault TA. Mammalian tissue oxygen levels modulate iron-regulatory protein activities in vivo. Science. 2004;306(5704):2087-2090.

51. Allerson CR, Cazzola M, Rouault TA. Clinical severity and thermodynamic effects of iron-responsive element mutations in hereditary hyperferritinemia-cataract syndrome. J Biol Chem. 1999;274(37):26439-26447.

52. Percy MJ, et al. Is congenital secondary erythrocytosis/polycythemia caused by activating mutations within the HIF-2 alpha iron-responsive element? Blood. 2007;110(7):2776-2777.

53. LaVaute $\mathrm{T}$, et al. Targeted deletion of the gene encoding iron regulatory protein- 2 causes misregulation of iron metabolism and neurodegenerative disease in mice. Nat Genet. 2001;27(2):209-214. 